\title{
DTLS improvements for fast handshake and bigger payload in constrained environments
}

\author{
Philippe Pittoli, Pierre David, Thomas Noël \\ ICube, Université de Strasbourg, France
}

\begin{abstract}
Transport Layer Security (TLS) is a protocol defined by the IETF to secure communications on the Internet, and Datagram Transport Layer Security (DTLS) is its version based on UDP. DTLS is the proposed solution to secure the Internet of Things (IoT). As IoT devices are constrained in memory, in code size and in computation speed, DTLS overhead is a crucial parameter for communication efficiency. The contribution presented in this paper is an improved version of DTLS, with fewer handshake messages and a reduced payload overhead, without compromising security. Fewer handshake messages means a reduced connection delay, with 6 signalling packets instead of 10. Reducing payload overhead improves communication latency and provides more room for application data. As such, our work provides a more efficient connectionbased security protocol for the IoT domain.
\end{abstract}

\section{Introduction}

Constrained environments are used in a wide range of situations, varying from autonomous wireless sensors for environmental studies to industrial systems with energy supplied devices. Network technologies are equally very diversified, from the multi-hop or single hop wireless network based on IEEE 802.15.4 to a wired network such as Ethernet in industrial context. However, even with such a diversity, there is always a need for end-to-end security in order to protect data from illegitimate eavesdroppers and to protect devices from malicious intrusions.

TLS (Transport Layer Security) is a fundamental cryptographic protocol whose main purpose is to establish a secured connection between two applications. This is accomplished via a negotiation of cryptographic material, enabling the peers to encrypt the whole communication with a shared encryption key. TLS is utilized in various ways on the Internet, to protect web traffic (HTTPS), to secure the e-mail transport protocol (SMTP/TLS) and so on. However, the TCP connection required by TLS is costly, specially in constrained environments such as the Internet of Things (IoT).

Datagram Transport Layer Security (DTLS) [RM12] is a modified version of the TLS protocol to use UDP as the underlying layer. It has been designed for purposes such as real-time applications. The IETF promotes its use on constrained devices that are utilized in the emerging wireless technologies such as Internet of Things (IoT). 
Despite the use of UDP, DTLS is still costly for two main reasons. First, the number of messages transmitted during the handshake delays the first data packet (which is emphasized in duty cycled network $\left[\mathrm{VTW}^{+} 15\right]$ ) and energy consumption. Second, the DTLS header limits the room available for application data in a packet.

This article presents an improved version of DTLS for the sake of speed, allowing quicker connection establishments. We reduce the amount of signalling packets and the header overhead, using IETF recommendations as a basis. In order to concentrate on the DTLS layer only, we do not make any assumption on other layers. As a consequence, we reduce the whole protocol stack to the bare minimum as shown in Figure 1: neither IP nor transport layer nor application layer, in order to measure only the impact of DTLS over a communication network based on IEEE 802.15.4.

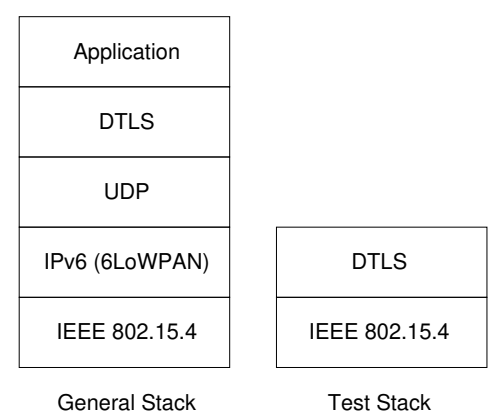

Fig. 1. Classic protocol stack as in deployed networks, and our protocol stack to only measure the impact of the DTLS protocol in communications.

As encryption speed is an important parameter in security protocol efficiency, we quantify in this paper the gain of using hardware encryption rather than software encryption.

We compare our proposal to the original DTLS protocol, and we also compare the software and hardware encryptions in order to measure the improvement of communication speed with a specialized hardware. We focus on the connection speed (handshake and application data exchange) and the memory (RAM and Flash) consumption.

The rest of this paper is structured as follows. In Section 2, we introduce DTLS principles. Section 3 describes the objective of this article, and the choice of algorithms. We then explain our improvements over the DTLS protocol in Sections 4 and 5. Experimental study of performances has been conducted. We describe our hardware platform in Section 6, and we summarize performance results in Section 8. We describe related works in Section 9, before concluding in Section 10. 


\section{Overview of DTLS}

The goal of DTLS is to secure a connection between two applications. It is composed of an algorithm and cryptographic material negotiation, then an application data exchange with encryption (confidentiality), integrity checking and signature (authenticity). DTLS is layered above UDP as the transport protocol, where TLS is layered above TCP. This difference implies that DTLS doesn't have a transport layer protocol that handles packet losses and retransmission, reordering and fragmentation itself. Figure 2a shows the different control packets exchanged during connection establishment, which are explained later in this section.

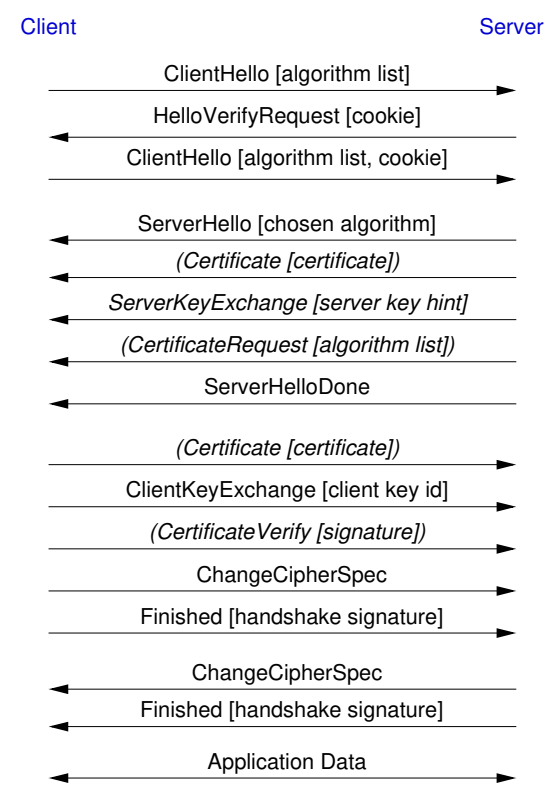

Fig. 2a. DTLS handshake. Messages in italics are optional. Payloads of messages are enclosed within square brackets. Context specific messages are enclosed within parentheses.

\subsection{Handshake}

In order to establish a handshake based on DTLS protocol, multiple packets need to be transmitted, as shown by figure 2a. First, the client sends a message Client Hello containing the list of algorithms supported by the client to manage the session, and a random number to avoid using the same cryptographic key 


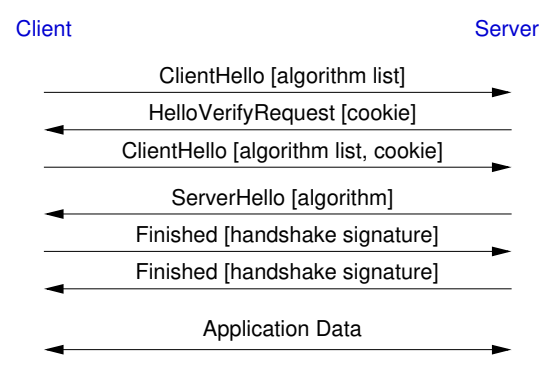

Fig. 2b. Optimized DTLS handshake.

to encrypt messages on every session. The server replies with a message Hello Verify Request containing a cookie which must be sent back by the client with a new message Client Hello. This mechanism is needed to mitigate a deny of service attack targetted to overload the server memory by creating multiple sessions. With this mechanism, the server does not keep a state between the first and the second client messages.

Then the server sends a Server Hello message with the list of algorithms to use to enforce the integrity, the confidentiality and the authentication, based on the list previously sent by the client. Three optional messages come after: a certificate to authenticate the server to the client, its identity is sent with a Server Key Exchange message to help the client choosing the right encryption key, and a message Certificate Request if the server requires a certificate to authenticate the client. To conclude this phase, the server sends a message Server Hello Done.

If required, the client may send its certificate with a Certificate message. Next, it sends a Client Key Exchange to help the server choose the appropriate encryption key and then it may send a certificate verification with a Certificate Verify message.

Finally the client, then the server, send two messages. The first one, Change Cipher Spec, indicates to the peer that the next messages will be protected with the previously negotiated algorithms and cryptographic material. The second message, Finished, includes a signature of the whole handshake, to ensure that the messages had not been modified during the connection phase.

Messages within parentheses in Figure 2a are sent only if the certificate system is needed. The Pre-Shared Key (PSK) mechanism, which assumes that authentication is performed by the knowledge of the pre-shared key, does not involve any certificate.

\subsection{Application Data}

Once the handshake is completed, the session is up and payload data may be exchanged. The protocol used to carry the data is simple: it only encrypts, signs and verifies the integrity. 


\section{IoT application: key sharing, encryption, certificates}

Our main objective will be to be able to establish a secure connection as quickly as possible with a very constrained hardware (from $8 \mathrm{MHz}$ processor) with a constrained link layer (IEEE 802.15.4). To that end we will select the appropriate algorithms and mechanisms, for the key sharing, the encryption, and so on. Choices made for very constrained environments will still be valid for less constrained ones.

There are several ways to share encryption keys. The simplest is to share the key with the devices before the deployment or with an out-of-band communication. This can be acceptable in a sensor network. Sharing encryption keys with a certificate system is a costly mechanism, even with elliptic curves [LN08]: on a device with a $13 \mathrm{MHz}$ processor, there is more than one second to initialize, sign and verify a certificate with resources consuming optimisations (about 1500 bytes of RAM and 10 to $15 \mathrm{~KB}$ of Flash memory), and several seconds for a $8 \mathrm{MHz}$ processor.

At the IETF, the DICE working group has defined a DTLS profile suitable for IoT applications [TF15]. It is reasonably implementable on many constrained devices in terms of algorithm complexity and speed. The profile recommends the use of Counter with CBC-MAC (CCM) encryption method, with 8 bytes of Message Authentication Code (MAC) and the AES-128 symmetric encryption algorithm. For key sharing, the profile recommends the use of pre-shared keys or, if possible, Diffie-Hellman exchange for the key agreeing with a certificate authentication system optimized with elliptic curves (Elliptic Curves Digital Signature Algorithm).

\section{Improvement: handshake message removal}

As shown with Figure 2a, DTLS is a verbose protocol which was not intended to be used in very constrained networks. In this section, we propose to improve the DTLS handshake by reducing the total number of packets exchanged in preshared key (PSK) communication context. We follow the recommendations of the DICE working group for the encryption mechanism: we use AES 128 and CCM 8, and we pre-share the cryptographic keys. We do not use Diffie-Hellman nor certificates because they are costly even with elliptic curves. Thus, the messages required by the certificate system as shown in Figure 2a are removed. Next, we propose to remove handshake messages which are for signalisation only and that do not contain any useful data (shown by the messages in square brackets): it is possible to do so because we fix the messages sequence, which implies that to indicate the end of a message sequence with a signalling packet becomes non essential. We also remove messages which indicate the identity of a peer, because it is possible to deduce this piece of information in several ways: with a sub-layer protocol (by the MAC or IP address for instance), or from the upper application protocol.

It is important to note that even if the MAC (or IP) addresses are used to identify a peer, it is not possible for an attacker to get any useful data: the 
attacker may spoof an address, but if it does not know the cryptographic keys, as they are never sent in clear text, the connection cannot be established.

Figure $2 \mathrm{~b}$ summarizes our proposed DTLS optimizations.

ServerHelloDone and ChangeCipherSpec : these messages are removed because they do not contain any useful data. The ServerHelloDone message indicates the end of a message sequence from the server to the client but, in our DTLS version, the server has to send only one message (Server Hello) in this sequence. We can further notice that the ChangeCipherSpec is about to be removed in TLS 1.3 [Res15].

ServerKeyExchange : this message is non essential if we have only one server and a single key is to use for the server at a time, or if the client already knows the server identity [ET05] (or it can be deduced) and there is only one key at a time.

ClientKeyExchange : this message may be removed in two cases: either the client is located one single hop away to the server, thus letting the server use the MAC (or IP) address to deduce the client identity, or the application protocol is used to communicate the client identity to the server.

With these optimizations, we remove up to five messages during the handshake and settle the number of exchanged messages (no more optional message left). This simplifies the final code, and it is expected to lower the binary size, implementation errors, the maintenance and improve the readability.

The counterpart of these optimizations is that they remove compatibility with current DTLS implementations.

\section{Improvements: redundancy during the application data exchange}

Once the connection is established, DTLS transmits the application data using two layers (Record and Application layers) whose headers are shown in figure 3. The Record part contains two fields (Epoch and Sequence Number) used to detect packet losses, duplication and unordered packet arrival. When Authenticated Encryption with Associated Data (AEAD) algorithm is used to secure the communication, the Application Data part contains both the payload and an Initialization Vector (IV). The IV has to be unique on every message and is conventionally composed of the Epoch and the Sequence Number from the Record part.

We can collapse this redundant information ( 8 bytes) in the two layers, reducing the DTLS communication overhead from 29 to 21 bytes. This 8-byte redundancy represents $6 \%$ of the total payload over a IEEE 802.15.4 connection. 


\section{Record Layer}

\begin{tabular}{|l|l|l|l|l|}
\hline CT & Version & Epoch & Sequence Number & MSize \\
\hline
\end{tabular}

Application Layer

\begin{tabular}{|l|l|l|}
\hline Epoch & Sequence Number & Payload ... \\
\hline
\end{tabular}

Fig. 3. Redundancy in the headers, due to a conventional usage of the initialization vector in AEAD encryption.

The gain is especially the higher packet payload available (97 bytes instead of 89 when used on a IEEE 802.15.4 network, hence 8.2 \%). Applications have more room to carry data on a single fly. In addition, the removal of this redundancy is expected to lower the transmission duration.

The counterpart of this optimization is that it removes compatibility with current DTLS implementations.

\section{Experiment environment}

We run an experiment in order to measure the improvements brought by our solution in term of memory consumption (RAM and Flash), and connection speed (both handshake and application data exchange).

In this section, we describe the hardware and software environment of our experiment (section 8). Our code is based on TinyDTLS which is a library that implements the current latest DTLS protocol version (1.2).

\subsection{Hardware platform}

Our hardware platform is based on ATMega128RFA1 microcontroller (Zigduino) with a native IEEE 802.15.4 connectivity, $16 \mathrm{kB}$ RAM, $128 \mathrm{kB}$ Flash and a $16 \mathrm{MHz}$ CPU frequency. The 802.15.4 connectivity is set in peer-to-peer mode, radio always on in order to avoid the wake-up delay for the link layer. There is only one hop from the client to the server.

The first device acts as a DTLS server which listens to the network, the second acts as a DTLS client which periodically sends a message with an arbitrary length.

\subsection{IEEE 802.15.4 and CSMA-CA}

To explain the time taken by a packet to reach its destination, we need to know the waiting duration before the transmission. These pieces of information are available from the IEEE 802.15.4 standard [IEE06]. The time spent waiting for CSMA-CA (expressed in symbols) is described with equation 1. BE means Backoff Exponent, it is used to fix an upper bound to the waiting time of the CSMA-CA algorithm. 


$$
T_{\text {wait }_{\text {symbols }}}=\operatorname{random}\left(2^{\mathrm{BE}}-1\right) * \text { unit backoff period }
$$

This equation leads to the maximum time spent for CSMA-CA in equation 2 .

$$
\begin{aligned}
T_{\text {wait }} & =\frac{T_{\text {wait }_{\text {symbols }}}}{\text { nb symbols per second }} \\
& =\frac{\text { random }\left(2^{3}-1\right) * 20}{62500} \\
& \leq 2.24 \mathrm{~ms}
\end{aligned}
$$

The maximum waiting time on the first try when we want to send a message is $2.24 \mathrm{~ms}$ (equation 2), with $B E=3$ which is the best case (first attempt to access the medium) and the backoff period is 20 (physical unit). If the random function follows a linear distribution, then the average waiting time will be 1.12 ms before sending a message (without interferences).

The minimum link layer has 10 bytes, but we instead use 12 bytes because we add the Personal Address Network identification (PAN id) in addition to the four bytes of MAC addressing (source, destination), in order to accomplish experiments in our laboratory with different sub-networks.

\subsection{Network and transport layers}

As explained in section 1, we do not use network or transport layer since we want to focus on pure DTLS performances and to avoid any unneeded overhead. Network and transport layers are not required for a single hop network since every node in the local network can reach each other, so the addressing is done with the MAC header alone.

\section{Hardware encryption}

Since the IEEE 802.15.4 standard requires an AES encryption to secure the communications on the link layer, the AES hardware encryption may be available on many of the IEEE 802.15.4 enabled devices. Dedicated hardware (with specialized instructions) improves an algorithm speed. Table 1 shows the results of a preliminary test of hardware encryption on our microcontroller. The encryption of 16 bytes with AES-128 on specialized hardware is five times quicker than software encryption. The CCM encryption mechanism advised by the DICE profile uses AES intensively, it is interesting to observe the practical gain in connection establishment and transmission durations. 
Table 1. Comparison between software and hardware AES encryptions on a 16 bytes block.

\begin{tabular}{lll}
\hline & $\begin{array}{l}\text { Software } \\
\text { Encryption }\end{array}$ & $\begin{array}{l}\text { Hardware } \\
\text { Encryption }\end{array}$ \\
\hline 16 bytes & $484 \mu \mathrm{s}$ & $96 \mu \mathrm{s}$ \\
\hline
\end{tabular}

\section{Measured performances}

To measure the message exchange duration between two devices (or applications) without reliable time synchronisation, we run an experiment by sending a message from a device to another, then sending it back. The message sizes used are 1 byte (the shortest possible message, such as a temperature without further application protocol like CoAP) and 87 bytes (the largest message on IEEE 802.15.4 with a 12-byte header).

Our experiment is performed with several configurations. The first one is a simple port of DTLS over ATmega128RFA1, the second one is our optimized DTLS exposed in sections 4 and 5, the next two are vanilla DTLS and our optimized DTLS with hardware encryption, and the final one is a non-secure exchange (without DTLS nor encryption). The last configuration aims at putting the results into perspective and knowing the cost of security.

\subsection{Flash memory and RAM}

Table 2. Comparison between DTLS, optimized DTLS, their hardware encryption variant and without DTLS (nor encryption)

\begin{tabular}{llllll}
\hline & DTLS & $\begin{array}{l}\text { Optimized } \\
\text { DTLS }\end{array}$ & $\begin{array}{l}\text { DTLS } \\
\text { H.E. }\end{array}$ & $\begin{array}{l}\text { Optimized } \\
\text { DTLS H.E. Security }\end{array}$ \\
\hline RAM (bytes) & 11216 & 10388 & 7120 & 6292 & 897 \\
Flash (bytes) & 48966 & 46610 & 42208 & 39852 & 8000 \\
median handshake dur. $(\mu \mathrm{s})$ & 233214 & 186660 & 220840 & 173628 & \\
median dur. (1 byte) $(\mu \mathrm{s})$ & 18720 & 18248 & 11220 & 10748 & 4780 \\
median dur. (87 bytes) $(\mu \mathrm{s})$ & 43664 & 43664 & 21488 & 21168 & 10600 \\
\hline
\end{tabular}

Table 2 summarizes the observed consumption results. First, the cost in RAM for the security is more than $10 \mathrm{kB}$, and almost $41 \mathrm{kB}$ in Flash. The optimized version consumes less of these ressources ( $7.3 \%$ less RAM and 5\% less Flash), which is explained by the code removal due to the unneeded messages. The biggest difference is between software and hardware encryption: the encryption library (from OpenBSD) is speed-optimized by unrolling loops which implies a 
larger amount of instructions. On the other hand, the AES algorithm is removed with hardware encryption, which reduces the binary size of $4 \mathrm{kB}$, it is useful when the application code is large and this should be considered for complex applications over very constrained devices.

\subsection{Handshake performances}

The handshake duration is measured on the client, from the first message of the connection to the handshake final (Finished) message. The median of observed handshake durations done over 100 connections is about $233214 \mu$ s with DTLS, and $186660 \mu$ s with our optimized version of DTLS. The gain is $46.5 \mathrm{~ms}, 19.9 \%$ of the duration of the handshake: the message removal lowers the number of exchanges, thus lower the total duration of CSMA-CA waiting.

\subsection{Data exchange performances}

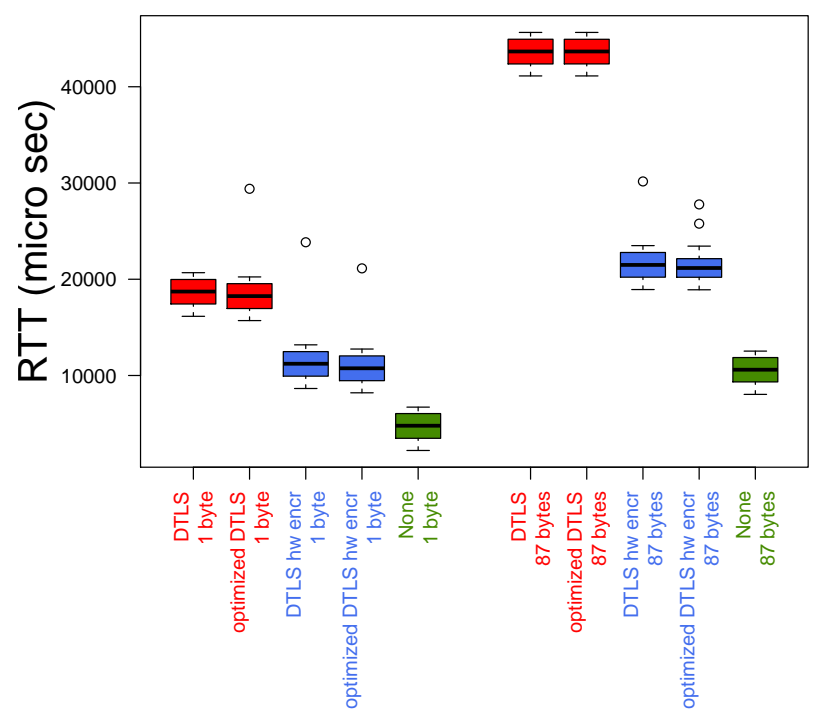

Fig. 4. Sending a message and returning it back, with only one byte then 87 bytes message: DTLS, optimized DTLS, then their hardware encrypted versions and without DTLS.

The removal of the Initialization Vector (IV) explained in section 5 brings several improvements: transmission is quicker because the message is smaller, leaving more room for application data and decreasing the use of fragmentation. 
Figure 4 shows our results over 3000 measurements for each configuration. The difference between DTLS and our optimized version is pronounced with the single byte message because difference of the message size to transmit is proportionally more important, the gain is about $2.6 \%$. The difference between DTLS (optimized or not) and a non protected exchange is measured with a non-secure exchange, to put the cost of security into perspective. The message protection implies a RTT 3.9 times higher than without protection. Encryption, plus the overhead in the packet payload for DTLS, significantly slows down the connection.

Secondly, the message payload can be 8 bytes higher, thus the number of messages required for sending a certain amount of data is lower. For instance to send 1700 bytes, which represents 18 messages with the optimized version of DTLS, it is required to send 20 messages of the original DTLS.

The different improvements can be meaningful depending on the application. For instance, the handshake optimization lowers the connection duration: this helps connection with highly mobile nodes (drones, cars, etc.). There is only a slight improvement on the latency (on small packets) due to the IV removal, so it is useful mainly to reduce fragmentation.

\section{Related Work}

Lithe $\left[\mathrm{RSH}^{+} 13\right]$ takes another approach to reduce DTLS overhead: it relies upon 6LoWPAN to compress DTLS informations in order to gain up to 8 bytes during data exchange in the optimal case, where Epoch and Sequence Number fields (in the Record header) are truncated respectively to 8 and 16 bits (instead of 16 and 48 bits). This reduced width limits the number of exchanged messages in a single connection. Lithe does not remove redundant informations between the Record and Application layers. Furthermore, Lithe is tied to 6LoWPAN and is not independant from the lower layers.

Robust Header Compression (ROHC) is a method described in several RFCs to compress headers, such as IP and UDP ones (amongst others). The key principle is not to send an header if it is the same than the previous one, or just to send the difference. This may be used to limit the protocol payload, and can be used with our optimized DTLS if the IP context can't be avoided.

Elliptic curves cryptographic performances on sensors are tested on [LN08] through a number of evaluations with a library called TinyECC. This library lets the developers turn on and off several optimizations of elliptic curves algorithms, and can be used to improve the speed performances and the power consumption. Thanks to this work, we concluded that elliptic curves are one order of magnitude slower than symmetric cryptography on constrained devices.

Finally, the number of messages exchanged during the handshake induces degraded performance in duty-cycled networks, this is covered by $\left[\mathrm{VTW}^{+} 15\right]$. In

those networks the handshake duration can be more than 30 seconds, but our optimization can greatly helps to boost the performances. 


\section{Conclusion and Future Work}

Our work leads to an improvement of the time spent on the handshake, of the RAM and Flash memories used by removing several messages. The most significant improvement is the larger payload of application data, due to the removal of Initialization Vector for AEAD algorithms, such as suggested by the DICE IETF working group. This improvement is at the cost of an incompatibility with current DTLS implementations.

As future work, we could experiment new standardized algorithms for encryption (ChaCha20) and for integrity checking (Poly1305) to see the differences in memory, code size and processing time. Furthermore, the IETF working group on TLS 1.3 tends to change completely the protocol, leading to a simpler and faster handshake, new mandatory-to-implement algorithms and to even change the fields.

\section{References}

[CCCP15] A. Capossele, V. Cervo, G. De Cicco, and C. Petrioli. Security as a coap resource: an optimized dtls implementation for the iot. 2015.

[ET05] P. Eronen and H. Tschofenig. Pre-Shared Key Ciphersuites for Transport Layer Security (TLS). RFC 4279 (Proposed Standard), December 2005.

$\left[\mathrm{HGmH}^{+}\right]$Tobias Heer, Oscar Garcia-morchon, Rene Hummen, Sye Loong Keoh, Eep S. Kumar, and Klaus Wehrle. Security Challenges in the IP-based Internet of Things. Wirel. Pers. Commun, pages 527-542.

[IEE06] IEEE. 802.15.4, 2006. http://standards.ieee.org/getieee802/download/802.15.42006.pdf.

[LN08] A. Liu and P. Ning. TinyECC: A configurable library for elliptic curve cryptography in wireless sensor networks. In Proceedings of the 7th International Conference on Information Processing in Sensor Networks, IPSN '08, pages 245-256, Washington, DC, USA, 2008. IEEE Computer Society.

[Res15] E. Rescorla. The Transport Layer Security (TLS) Protocol Version 1.3. Internet-Draft draft-ietf-tls-tls13-07.txt, IETF Secretariat, July 2015.

[RM12] E. Rescorla and N. Modadugu. Datagram Transport Layer Security Version 1.2. RFC 6347 (Proposed Standard), January 2012.

$\left[\mathrm{RSH}^{+} 13\right]$ Shahid Raza, Hossein Shafagh, Kasun Hewage, Hummen Rene, and Thiemo Voigt. Lithe: Lightweight Secure CoAP for the Internet of Things. IEEE Sensors Journal, 13(10):3711-3720, 2013.

[TF15] Hannes Tschofenig and Thomas Fossati. TLS/DTLS Profiles for the Internet of Things. Internet-Draft draft-ietf-dice-profile-17.txt, IETF Secretariat, October 2015.

$\left[\mathrm{VTW}^{+} 15\right]$ Mališa Vučinić, Bernard Tourancheau, Thomas Watteyne, Franck Rousseau, Andrzej Duda, Roberto Guizzetti, and Laurent Damon. DTLS Performance in Duty-Cycled Networks. In International Symposium on Personal, Indoor and Mobile Radio Communications (PIMRC - 2015), Hong-Kong, China, aug 2015. IEEE. 